\title{
COMPARAÇÃO DE MODELOS NÃO-LINEARES PARA DESCREVER O CRESCIMENTO DE FÊMEAS DA RAÇA GUZERÁ 1
}

\author{
HENRIQUE NUNES DE OLIVEIRA ${ }^{2}$, RAYSILDO BARBOSA LÔBO ${ }^{3}$ e CARMEN SILVA PEREIRA ${ }^{4}$
}

\begin{abstract}
RESUMO - Dados de crescimento e reprodução de 573 vacas da raça Guzerá, nascidas entre 1961 e 1985, na Fazenda Canoas, em Curvelo, MG, foram analisados com o objetivo de estabelecer um padrão médio de crescimento, mediante o uso de um modelo matemático que se ajuste adequadamente aos dados. Os modelos Brody, Bertalanffy, Logístico, Gompertz e Richards foram ajustados aos dados de peso/idade, coletados até 1992, e comparados quanto à qualidade de ajustamento. Os pesos assintóticos e as taxas de maturidade estimadas foram, respectivamente: para o modelo Brody, 464,49 e 0,046; para o Bertalanffy, 453,18 e 0,065; para o Logístico, 447,05 e 0,085; para o Gompertz, 449,89 e 0,075, e para o Richards, 458,26 e 0,055. O modelo Richards apresentou dificuldades computacionais para ajustamento aos dados. Os outros modelos se revelaram adequados para descrever o crescimento nesses animais, apresentando pequenas variações na qualidade de ajustamento, de acordo com os critérios utilizados. O modelo Bertalanffy foi escolhido para representar a curva média de crescimento dos animais, por apresentar um ajustamento superior no conjunto dos critérios.
\end{abstract}

Termos para indexação: peso, maturação, taxa de crescimento, modelo matemático.

\section{COMPARISON OF NON-LINEAR MODELS FOR DESCRIBING GROWTH OF GUZERAT BEEF CATTLE FEMALES}

\begin{abstract}
Data on growth and reproduction of 573 females Guzerat beef cattle born between 1961 and 1985, at Fazenda Canoas, located in the county of Curvelo, MG, Brazil, were used to obtain a growth pattern for the breed, through the use of a mathematical model. Weight-age data, collected up to 1992, were used to fit five nonlinear models: Brody, Bertalanffy, Logistic, Gompertz, and Richard. Estimated assyntotic weight and growth rate were, respectively, for Brody model 464.49 and 0.046 ; for Bertalanffy model 453.18 and 0.065 ; for Logistic model 447.05 and 0.085 ; for Gompertz model 449.89 and 0.075 , and for Richards model 458.26 and 0.055 . Richards model presented computational difficulty to fit the data. Other models showed good fit to the data, and presented little variations on the criteria chosen. Bertalanffy model showed a better goodness of fit on the set of criteria and was the choice to describe the growth patterns of Guzerat cattle.
\end{abstract}

Index terms: weight, maturation, growth rate, mathematical models.

${ }^{1}$ Aceito para publicação em 15 de março de 2000.

Extraído da tese de doutorado do primeiro autor apresentada à Faculdade de Medicina de Ribeirão Preto (FMRP), USP.

${ }^{2}$ Méd. Vet., Dr., Prof. Assistente, Dep. de Melhoramento e Nutrição Animal, Universidade Estadual Paulista (UNESP), Caixa Postal 560, CEP 18618-000 Botucatu, SP. E-mail: hnunes@fca.unesp.br

${ }^{3}$ Méd. Vet., Dr., Prof. Adjunto, Dep. de Genética, FMRP, USP, Av. Bandeirantes, 3900, CEP 14409-900 Ribeirão Preto, SP. E-mail: raylobo@genbov.fmrp.usp.br

${ }^{4}$ Eng. Agrôn., Dra, Prof. Adjunta, Dep. de Zootecnia, Escola de Veterinária, Universidade Federal de Minas Gerais (UFMG), Caixa Postal 567, CEP 30161-970 Belo Horizonte, MG. E-mail: carmensp@ufmg.br

\section{INTRODUÇÃO}

Com base em pesquisas que enfocam fases iniciais de crescimento, os zebuínos têm sido classificados como animais tardios, em comparação com os bovinos de origem européia (Pereira, 1983). Fica implícito, nessa classificação, que os zebuínos crescem lentamente nas etapas iniciais da vida, mas devem atingir tamanhos comparáveis aos taurinos, quando adultos. Contudo, são praticamente inexistentes, no Brasil, estudos com pesos de todo o ciclo de vida dos zebuínos, desde o nascimento até a maturidade, e portanto não existe uma descrição glo- 
bal do crescimento nem foi estabelecido o que possa ser considerado como padrão médio de crescimento para zebuínos.

Modelos matemáticos não-lineares, desenvolvidos empiricamente para relacionar peso e idade, têm-se mostrado adequados para descrever a curva de crescimento. Esses modelos permitem que conjuntos de informações em séries de peso por idade, sejam condensados num pequeno número de parâmetros, para facilitar a interpretação e o entendimento do fenômeno.

Cinco desses modelos têm sido mais utilizados em bovinos. São eles: Brody (Brody, 1945), Bertalanffy (Bertalanffy, 1957), Richards (Richards, 1959), Logístico (Nelder, 1961) e Gompertz (Laird, 1965). Pode-se considerar que está bem estabelecido o conhecimento sobre seu uso para descrever o crescimento de fêmeas em algumas raças taurinas em ambientes de clima temperado (Brown et al., 1976; Perotto et al., 1992). Geralmente, os modelos Richards e Brody mostraram-se os mais adequados para descrição do crescimento nessas raças; o primeiro deles apresenta ligeira vantagem, em termos de qualidade de ajustamento aos conjuntos de dados, mas por outro lado, apresenta dificuldades computacionais para o ajustamento.

Os trabalhos que compararam esses modelos em zebuínos no Brasil utilizaram dados de animais cujas pesagens foram interrompidas antes que a maturidade fosse atingida (Cortarelli, 1973; Duarte, 1975; Ludwig, 1977). Desta forma, não se conhece o comportamento desses modelos em fases mais avançadas do crescimento, e nem qual deles seria mais adequado para descrição do crescimento total dos zebuínos.

Este trabalho teve o objetivo de estabelecer um padrão médio de crescimento de fêmeas da raça Guzerá, por um modelo matemático que se ajuste adequadamente aos dados.

\section{MATERIAL E MÉTODOS}

Os dados utilizados neste trabalho são referentes a 573 vacas da raça Guzerá nascidas na Fazenda Canoas, no município de Curvelo, MG, entre os anos de 1961 e 1985, filhas de 66 touros.
O clima da região é característico das zonas de cerrados, apresentando período seco correspondente ao outono e inverno, com temperaturas mais baixas nos meses de junho e julho, e $80 \%$ das chuvas, de outubro a março.

As pastagens da propriedade consistiam principalmente de capim-gordura (Melinis minutiflora, Pal. de Beauv.), braquiária (Brachiaria decumbens) e capim-jaraguá (Hyparrhenia rufa, Stapf). Todos os animais recebiam suplementação mineral a vontade, no cocho, o ano todo. Durante a estação chuvosa, o rebanho permanecia em regime de pasto. Na seca, os animais eram supridos de canade-açúcar, capim-napier verde, picado, e silagem de milho ou sorgo. As vacas em lactação e as novilhas em crescimento recebiam, ainda, suplementação parcial de concentrado, enquanto os touros e as vacas secas permaneciam no pasto. Os bezerros eram desmamados durante a quinzena que se seguia à data em que completavam oito meses de idade.

O regime de cobrição era no pasto, com duas estações de acasalamento, ou seja: a primeira, de julho a dezembro, e a segunda, de março a abril. Entretanto, durante o período a que se referem os dados, houve fases em que não foi adotada a estação de acasalamento, e outras em que a primeira estação foi mais longa.

Os animais eram pesados ao nascer e na primeira semana de todos os meses pares, até completarem 36 meses de idade; a partir de então, e enquanto permaneciam no rebanho, os animais eram pesados de quatro em quatro meses. A escolha dos animais jovens que deviam permanecer no rebanho era feita levando-se em conta as características raciais, a conformação, e o peso à desmama.

Os dados eram obtidos das fichas individuais de registro zootécnico atualizadas, até o ano de 1992.

\section{Ajustamentos dos modelos aos dados}

As análises dos dados foram desenvolvidas em computadores do Setor de Computação do Departamento de Genética da Faculdade de Medicina de Ribeirão Preto USP, e no Instituto de Física de São Carlos - USP.

Os modelos utilizados para descrever o crescimento dos animais foram: Brody, Bertalanffy, Richards, Logístico, e Gompertz. A parametrização dos modelos foi a mesma utilizada por Brown et al. (1976), e está apresentada na Tabela 1.

Os modelos foram ajustados às séries de peso por idade de cada fêmea, individualmente, de acordo com o método de Gauss-Newton modificado, descrito por Hartley (1961) para modelos não-lineares. Foi utilizado o procedimento "NLIN" do Sistema SAS (SAS Institute, 1985) para obtenção das estimativas individuais dos parâmetros e dos parâmetros médios a partir da totalidade dos dados disponíveis. 


\section{Comparação dos modelos quanto ao seu ajustamento aos dados}

A comparação entre modelos quanto ao seu ajustamento aos dados, quando não existe um modelo verdadeiro, pressupõe o teste da hipótese nula de que todos os modelos são igualmente bons para este fim, contra a hipótese alternativa de que um ou mais deles são melhores que os outros. Embora testes estatísticos objetivos para aceitar ou rejeitar a hipótese nula fossem desejáveis, a estrutura dos dados, a forma como foram analisados e a natureza complexa do processo descrito, dificilmente conduziriam a um teste definitivo. Desta forma, foram estabelecidos alguns critérios para comparação dos parâmetros, embora a escolha de um modelo como o melhor para descrever os dados fosse subjetiva, já que alguns desses critérios podem ser mais importantes que outros, dependendo da aplicação que terão os resultados.

A qualidade de ajustamento foi, inicialmente, avaliada por dois critérios: o quadrado médio do erro, e o coeficiente de determinação. As comparações entre as médias dos modelos, nesses dois critérios, foram feitas considerandose, para o cálculo das médias, apenas as observações em que a convergência foi atingida em todos os modelos. Outros dois critérios utilizados na comparação da qualidade de ajustamento foram obtidos em cada classe de idade. Em cada modelo, foram calculados o desvio médio entre o peso predito e o observado, e a correlação entre os dois. O primeiro desses critérios serve como indicativo da presença de vícios em fases distintas da vida do animal. A correlação entre o peso predito e o observado mostra como as variações dos pesos observados são representadas por variações nos pesos preditos a cada idade. Os pesos preditos utilizados no cálculo dos desvios e estimação das correlações foram obtidos em cada idade em que o animal foi pesado. As pesagens realizadas com idades entre $1 \mathrm{e}$ 2,99 meses foram agrupadas na classe de dois meses; as realizadas entre 3 e 4,99 meses foram agrupadas na classe de quatro meses, e assim sucessivamente.

TABELA 1. Parametrização dos modelos analisados.

\begin{tabular}{ll}
\hline Modelo & \multicolumn{1}{c}{ Equação } \\
\hline Brody & $\mathrm{y}_{\mathrm{t}}=\mathrm{A}\left(1-\mathrm{Be}^{-\mathrm{kt}}\right)$ \\
Bertalanffy & $\mathrm{y}_{\mathrm{t}}=\mathrm{A}\left(1-\mathrm{Be}^{-\mathrm{kt}}\right)^{3}$ \\
Logística & $\mathrm{y}_{\mathrm{t}}=\mathrm{A}\left(1+\mathrm{e}^{-\mathrm{kt}}\right)^{-\mathrm{M}}$ \\
Gompertz & $\mathrm{y}_{\mathrm{t}}=\mathrm{y}_{0} \mathrm{e}^{\mathrm{L}\left(1-\mathrm{e}^{-\mathrm{kt}}\right) / \mathrm{k}}$ \\
Richards & $\mathrm{y}_{\mathrm{t}}=\mathrm{A}\left(1-\mathrm{Be}^{-\mathrm{kt}}\right)^{\mathrm{M}}$ \\
\hline
\end{tabular}

${ }^{1} \mathrm{y}_{\mathrm{t}}$ : peso na idade t; A, B, k, L, m e $\mathrm{y}_{0}$ são os parâmetros ajustados.

\section{RESULTADOS E DISCUSSÃO}

\section{Estimação dos parâmetros dos modelos da curva de crescimento}

A amostra inicial, 573 fêmeas, incluiu 96 vacas descartadas antes de completarem 60 meses de idade e portanto não foram pesadas após essa idade. Considerando o trabalho de Morrow et al. (1978), segundo o qual, em gado europeu, estimativas precisas dos parâmetros dos modelos só podem ser obtidas em animais pesados até pelo menos 54 meses de idade, somente foram utilizadas informações de animais com pelo menos uma pesagem em idade superior a 60 meses.

A Tabela 2 mostra a amplitude de variação dos parâmetros da curva individual de crescimento estimados nos cinco modelos estudados, o número de animais nos quais o critério de convergência foi atingido em um número de iterações menor ou igual ao limite máximo estabelecido, e os parâmetros da curva ajustada conjuntamente em relação a todos os dados de todos os animais.

Quando se comparam as estimativas dos pesos assintóticos obtidas pelos vários modelos neste estudo, entre si, pode-se verificar que nos modelos Bertalanffy e Richards os valores foram um pouco abaixo dos valores do modelo Brody. Um pouco mais abaixo está o peso assintótico estimado pelo modelo Gompertz, enquanto no modelo Logístico o valor foi bem inferior. Esses resultados repetem as observações de Brown et al. (1976) e Perotto et al. (1992). Nesses estudos, o modelo Brody apresentou as maiores estimativas do peso assintótico, e o modelo Logístico, valores consistentemente abaixo dos demais. Goonewardene et al. (1981) também observaram que os modelos Logístico e Bertalanffy subestimaram o peso do animal adulto.

Na Fig. 1 estão representadas as curvas de crescimento formadas pelas médias dos pesos observados nas diferentes idades, e pelos pesos preditos em relação a essas mesmas idades a partir dos parâmetros estimados, com todos os dados de todas as fêmeas relativos aos modelos utilizados. Pode-se notar que no modelo Brody, embora o peso assintótico seja o mais alto entre os modelos, os pesos preditos na parte final da curva não parecem superestimar os pesos observados. Os pesos predi- 
tos pelo modelo Bertalanffy, na parte final da curva, estão abaixo dos pesos médios observados, o que indica que nessa fase os pesos são subestimados. Essa mesma tendência acentua-se muito no modelo Logístico e um pouco menos no modelo Gompertz. Nessa mesma fase, o comportamento do modelo Richards é mais próximo do comportamento do modelo Brody.

O parâmetro B representa o grau de maturidade do animal ao nascimento. Valores altos de B representam baixos graus de maturidade ao nascimento. Os valores de B encontrados neste trabalho, e apresentados na Tabela 2, são elevados, em comparação com os valores dos resultados citados na literatura. Entretanto, valores mais altos que os encontrados no presente trabalho são relatados quando a idade em que a primeira pesagem dos animais não foi tomada em época próxima ao nascimento. Alguns dos animais utilizados neste estudo não possuíam registro do peso ao nascimento, e embora a primeira pesagem nunca tenha excedido os três meses de idade, é possível que tal fato tenha contribuído para aumentar o valor médio encontrado.

Com base nas estimativas de A e B no modelo Brody, o peso médio predito em relação a esses animais seria, ao nascimento, de $13,46 \mathrm{~kg}$, enquanto a média dos pesos ao nascimento preditos a partir da curva individual de cada animal seria de $17,79 \mathrm{~kg}$. Desta forma, o modelo subestimou o peso ao nascimento, cuja média real foi de $27,3 \mathrm{~kg}$. Nadarajah et al. (1984) também observaram que o modelo Brody subestimava o peso ao nascimento. A média do parâmetro B referente ao modelo Bertalanffy está próxima das médias estimadas na maioria dos trabalhos em que este modelo foi utilizado. A variação de B neste modelo é limitada pela fixação de $\mathrm{M}=3$ (Brown et al., 1976). O peso ao nascimento, predito a partir da curva média, foi de 44,45 kg e 39,16 kg em relação à média das predições individuais. Da mesma forma, os modelos Logístico e Gompertz, com médias das predições individuais de peso ao nascimento de, respectivamente, $56,78 \mathrm{~kg}$ e 49,08 kg, superestimaram este peso. No modelo Richards, o peso predito a partir da curva média foi $29,42 \mathrm{~kg}$, enquanto a média dos pesos preditos individualmente foi de $29,06 \mathrm{~kg}$, bem próximas da média real. Tais resultados também estão de acordo com a literatura, na qual apenas o modelo Richards é citado como capaz de prever, com alguma precisão, os pesos dos animais nos estádios iniciais do crescimento pós-natal (Goonewardene et al., 1981).

O parâmetro k representa a taxa de maturidade do animal, e indica a velocidade de crescimento no sentido de atingir o peso assintótico, a partir de seu

TABELA 2. Número de animais em que o critério de convergência foi atingido, valores máximos e mínimos de cada parâmetro estimado nos ajustamentos individuais e parâmetros da curva média, estimada com uso de todas as pesagens de todas as fêmeas, de acordo com o modelo utilizado.

\begin{tabular}{lccccc}
\hline Modelo & Parâmetro & $\mathrm{N}$ & Máximo & Mínimo & Curva média \\
\hline Brody & $\mathrm{k}$ & 473 & 0,0906 & 0,0121 & 0,0461 \\
Brody & $\mathrm{B}$ & 473 & 1,3300 & 0,7694 & 0,9710 \\
Brody & $\mathrm{A}$ & 473 & 820,88 & 346,49 & 464,49 \\
Bertalanffy & $\mathrm{k}$ & 477 & 0,1361 & 0,02189 & 0,0652 \\
Bertalanffy & $\mathrm{B}$ & 477 & 0,9849 & 0,3348 & 0,5387 \\
Bertalanffy & $\mathrm{A}$ & 477 & 758,44 & 342,61 & 453,18 \\
Logístico & $\mathrm{M}$ & 477 & 5,2390 & 1,5756 & 2,8432 \\
Logístico & $\mathrm{k}$ & 477 & 0,1681 & 0,0371 & 0,0853 \\
Logístico & $\mathrm{A}$ & 477 & 673,03 & 338,85 & 447,05 \\
Gompertz & $\mathrm{k}$ & 477 & 0,15400 & 0,0297 & 0,0747 \\
Gompertz & $\mathrm{L}$ & 477 & 126,83 & 0,0518 & 0,1575 \\
Gompertz & yo & 477 & 15,655 & 7,2576 & 5,5506 \\
Richards & $\mathrm{M}$ & 314 & 0,1359 & 0,0138 & 0,038 \\
Richards & $\mathrm{K}$ & 314 & 1,0000 & 0,1368 & 0,0548 \\
Richards & $\mathrm{B}$ & 314 & 787,75 & 347,48 & 458,26 \\
Richards & $\mathrm{A}$ & 314 & & & \\
\hline
\end{tabular}



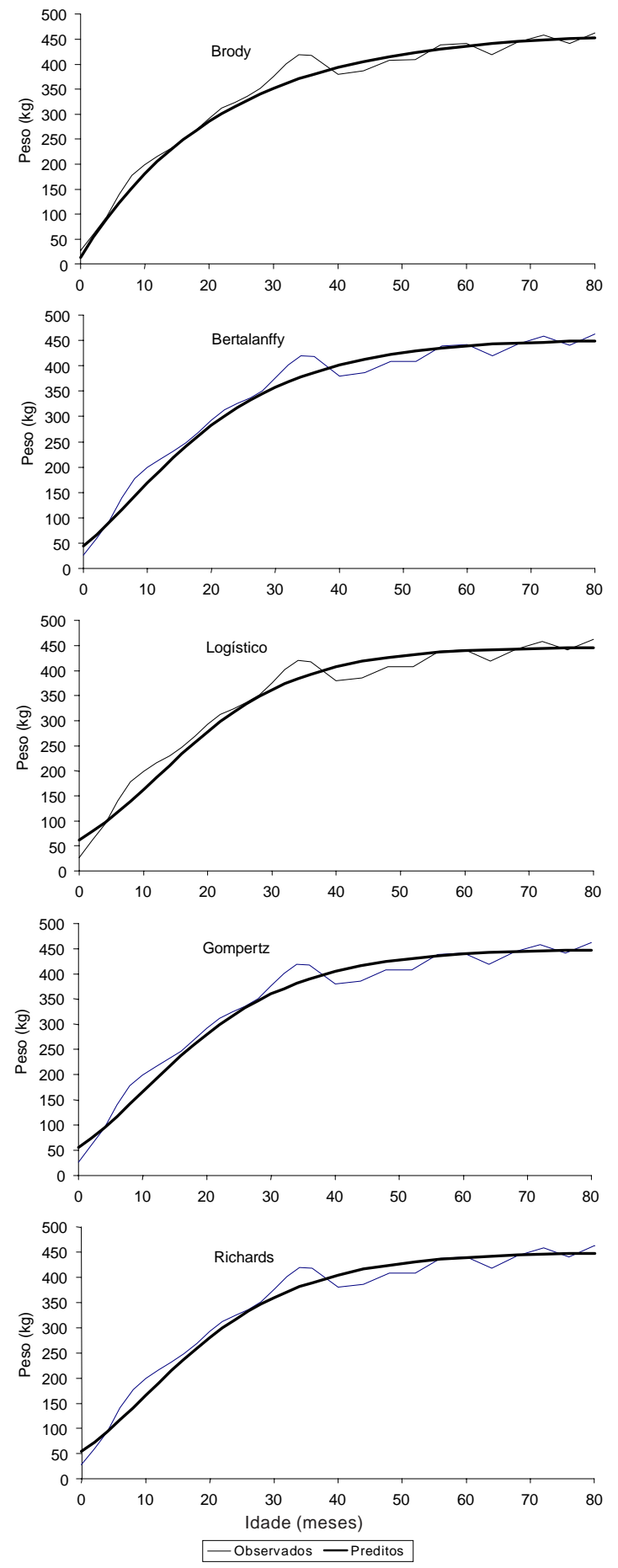

FIG. 1. Médias de pesos observados e pesos preditos em função da idade. Modelos Brody, Bertalanffy, Logístico, Gompertz e Richards. peso inicial. Quanto maior o valor de k, mais rapidamente o animal vai se aproximar do seu peso assintótico, ou seja, maior a velocidade de crescimento do animal no sentido de atingir o seu tamanho adulto. Animais com alto valor de $\mathrm{k}$ apresentam maturidade precoce, em comparação com indivíduos de valores mais baixos de $\mathrm{k}$ e de peso inicial semelhante. Com variação no peso inicial relativamente pequena, a variação entre os valores de k representa, com boa precisão, as variações na velocidade relativa com que o animal cresce.

Algumas informações importantes derivadas dos parâmetros da curva de crescimento foram sumariadas na Tabela 3. Para confecção desta tabela, cada dado foi obtido individualmente em cada animal, e posteriormente foi calculada a média.

Os modelos utilizados neste estudo não permitem estimar o ponto em que cessa o crescimento do animal, ou seja, o momento em que a maturidade é atingida. Geralmente, usa-se para tal fim, o ponto em que o animal atinge certo grau de maturidade arbitrariamente definido. Considerando-se como adultos animais com $95 \%$ do peso assintótico, a maturidade seria alcançada, pelo modelo Brody, aos 64 meses. Esse resultado aponta pouca precocidade do Guzerá em comparação com raças taurinas. Usando o mesmo modelo, Nadarajah et al. (1984) estimaram em 42 e 53 meses, respectivamente, a idade na qual fêmeas das raças Angus e Charolês atingiam esse grau de maturidade, e Beltran et al. (1992) observaram que fêmeas Angus de linha selecionada para maior peso à maturidade atingiam esse ponto em 56,4 meses . Mesmo o Zebu Gobra, estudado por Abassa (1987), chegou a $95 \%$ de maturidade com cerca de quatro meses menos que o Guzerá do presente estudo.

A idade em que os animais atingiram $95 \%$ do peso à maturidade variou consideravelmente, conforme $\mathrm{o}$ modelo empregado para sua predição; mas a idade em que os animais atingiram 50\% de maturidade foi praticamente a mesma em todos os modelos, conforme pode-se ver na Tabela 3. Esse resultado provavelmente reflete as diferenças que existem entre os modelos quanto ao ajustamento no final da curva. Os modelos que estimam os valores assintóticos mais altos também estimam as maiores idades para atingir a maturidade, e vice-versa. 
Os pontos de inflexão médios apresentados na Tabela 3 mostram também uma variação grande entre os modelos. Em nenhum deles o ponto de inflexão médio foi estimado dentro do intervalo em que o ganho observado foi máximo (entre quatro e seis meses de idade). Os valores mais próximos do intervalo foram obtidos nos modelos Richards (2,8 meses) e Bertalanffy (7,6 meses). Nos modelos Logístico e Gompertz, os pontos de inflexão estimados ocorreram próximo aos 12 meses de idade, correspondendo à curva dos ganhos de peso observados à fase de menor ganho entre o nascimento e os 20 meses. Apesar disto, quando se pensa que o ponto de inflexão estaria relacionado ao início da puberdade, esses modelos estariam mais perto da realidade.

\section{Comparação dos modelos quanto ao ajustamento aos dados}

Apenas 477 fêmeas que apresentavam pelo menos uma pesagem após a idade de 60 meses foram utilizadas para comparação dos modelos. A comparação foi realizada com base na observação de indicadores simples, sem aplicação de testes estatísticos, dada a natureza das variáveis utilizadas. Dificilmente, portanto, poder-se-ia chegar a uma conclusão definitiva quanto ao melhor modelo de acordo com cada um dos critérios, mas foi possível detectar falhas graves, e escolher um modelo, por apresentar melhor conjunto de resultados.
O modelo Richards foi o mais difícil de se ajustar aos dados, pois não atingiu o critério de convergência em 153 animais. O modelo Brody deixou de convergir em apenas quatro animais, e nos demais modelos a convergência foi atingida em todos os animais. As diferenças entre os modelos Brody, Bertalanffy, Logístico e Gompertz, quanto ao número de iterações, foram pequenas (Tabela 4). Esses resultados estão de acordo com a literatura, no que diz respeito ao modelo Richards (Duarte, 1975; Brown et al., 1976; Goonewardene et al., 1981; Denise \& Brinks, 1985; López de Torre et al., 1992; Perotto et al., 1992). Este modelo poderá ser usado para descrever a curva de crescimento em fêmeas zebuínas; entretanto, a dificuldade matemática na resolução do sistema exigirá um limite maior quanto ao número de iterações, ou um critério de convergência menos rigoroso.

As médias dos quadrados médios do erro e dos coeficientes de determinação, dos cinco modelos, estão na Tabela 4, que inclui apenas os animais que tiveram convergência alcançada nos cinco modelos $(n=314)$, para permitir melhor comparação entre esses. Os modelos Brody e Bertalanffy apresentaram média de quadrados médios do erro semelhante entre si, e menor que os demais modelos. As diferenças em coeficiente de determinação foram irrisórias e sem significado prático.

TABELA 3. Médias da idade e peso no ponto de inflexão, e idade com que os animais atingem $50 \%$ e $95 \%$ de maturidade, segundo os vários modelos utilizados.

\begin{tabular}{lccccc}
\hline Característica & Brody & Bertalanffy & Logístico & Gompertz & Richards \\
\hline Idade no ponto de inflexão (dias) & - & 231,07 & 371,76 & 311,05 & 84,54 \\
Peso no ponto de inflexão (kg) & - & 134,68 & 188,54 & 166,16 & 96,27 \\
Idade aos 50\% de maturidade (meses) & 14,48 & 14,69 & 14,99 & 14,17 & 14,09 \\
Idade aos 95\% de maturidade (meses) & 63,93 & 51,7 & 45,66 & 48,77 & 56,91 \\
\hline
\end{tabular}

TABELA 4. Valores médios dos indicadores sugeridos como critérios de comparação de curvas de crescimento, segundo o modelo adotado em relação aos 314 animais com solução convergente em todos os modelos.

\begin{tabular}{lrrrrr}
\hline Critério & Brody & Bertalanffy & Logístico & Gompertz & Richards \\
\hline Número de iterações & 8,33 & 8,95 & 9,43 & 10,31 & 17,02 \\
Quadrado médio do erro & 1465,54 & 1468,42 & 1518,23 & 1489,37 & 1488,22 \\
Coeficiente de determinação & 0,9899 & 0,9899 & 0,9895 & 0,9897 & 0,9901 \\
\hline
\end{tabular}


Esses resultados são diferentes da maioria dos resultados encontrados na literatura, em que os modelos Richards e Brody geralmente são apontados como os de melhor qualidade de ajustamento (Brown et al., 1976; Goonewardene et al., 1981). Dos trabalhos consultados, apenas o de López De Torre et al. (1992) considerou que o modelo Bertalanffy se ajustava tão bem aos dados de crescimento de fêmeas da raça Retinta quanto o modelo Brody. Esta raça, criada na Espanha, tem padrão de desenvolvimento um pouco diferente do padrão das raças normalmente criadas nos Estados Unidos e no Canadá, pois tem crescimento mais lento e reprodutivamente menos precoce. Assim, as diferenças entre este trabalho e os conduzidos nos Estados Unidos e Canadá talvez possam ser atribuídas aos padrões de crescimento dos animais estudados. A presença do ponto de inflexão e do pico devido à primeira cria, ambos, mais tarde, na vida do animal, quando em comparação com o gado de origem européia, podem constituir as principais causas de diferença.

A análise dos desvios médios e das correlações entre pesos observados e preditos, apresentados na Tabela 5, indicou que os cinco modelos têm padrão semelhante, super ou subestimando os pesos, em maior ou menor grau, nas mesmas idades, e representando melhor as variações dos pesos entre os seis e os 36 meses. Essa análise permitiu observar que os modelos Bertalanffy, Logístico e Gompertz apresentaram semelhança maior entre si quanto à magnitude dos valores, em comparação com os demais. Isto também pode ser observado pela inspeção visual dos gráficos, sendo notório o padrão semelhante dos modelos Bertalanffy, Logístico e Gompertz em contraste com os modelos Brody e Richards (Fig. 1).

Os desvios e as correlações mostraram que apenas o modelo Richards foi capaz de prever com alguma precisão o peso ao nascimento, conforme já haviam assinalado Goonewardene et al. (1981). Entre $2 \mathrm{e}$ 12 meses, os modelos Brody e Richards apresentaram menores desvios médios, o que indica vícios menores, além das maiores correlações, mostrando, assim, que as variações dos pesos são melhor representadas pelas variações observadas nas predições.

$\mathrm{Na}$ fase compreendida entre os 14 e 28 meses, as predições geralmente foram boas, tendo apresenta- do alta correlação com os pesos observados. Apesar de todos os modelos superestimarem, em maior ou menor grau, os pesos nessa fase, o modelo Brody apresentou os desvios mais acentuados, enquanto o modelo Richards apresentou os menores valores de correlação. Dos 30 aos 36 meses, os pesos preditos por todos os modelos subestimaram os pesos observados de forma acentuada, talvez em decorrência do início da vida reprodutiva, mas as correlações mostraram que as variações nos pesos reais foram bem representadas pelas variações nos pesos preditos em todos os modelos. O modelo Logístico apresentou os menores desvios e as maiores correlações. Após os 40 meses de idade, as variações verificadas nos pesos preditos representam bem menos as dos pesos observados. Isto poderia ser esperado, já que uma parcela considerável das variações nos pesos reais passa a ser decorrente do número de gestações e partos.

Em resumo, os modelos Brody, Richards e Logístico se alternaram, dependendo da fase considerada, como os melhores e os piores para representar os pesos observados. O modelo Gompertz apresenta valores bem próximos aos do modelo Logístico, enquanto o de Bertalanffy apresenta valores intermediários, e, embora em nenhuma das fases apresente a melhor predição, é o mais próximo do melhor, na maioria das fases.

Cada um dos modelos utilizados neste trabalho apresenta algumas vantagens e desvantagens em relação aos demais. O modelo Brody apresentou menor variância residual, mas os vícios entre os 14 e 36 meses de idade foram acentuados; o modelo Richards, aparentemente, não produziu vícios consideráveis em nenhuma fase do crescimento, mas apresentou dificuldades quanto ao aspecto computacional; os modelos Logístico e Gompertz superestimaram o peso ao nascimento, e aparentemente subestimaram o peso à maturidade; o modelo Bertalanffy apresentou variância residual bem perto da variância do modelo Brody, e não mostrou vícios consideráveis em nenhuma das fases, exceto no peso ao nascimento.

Desta forma, o modelo Bertalanffy parece o mais adequado para descrever a curva de crescimento na raça Guzerá, e pode-se indicar a curva descrita pelo modelo com os parâmetros encontrados na análise, 
TABELA 5. Correlações (r) e desvios médios (dm) entre pesos observados e preditos pelos cinco modelos estudados, de acordo com a idade, em meses.

\begin{tabular}{|c|c|c|c|c|c|c|c|c|c|c|}
\hline \multirow{3}{*}{$\begin{array}{c}\text { Idade } \\
\text { (meses) }\end{array}$} & \multicolumn{10}{|c|}{ Modelos } \\
\hline & \multicolumn{2}{|c|}{ Brody } & \multicolumn{2}{|c|}{ Bertalanffy } & \multicolumn{2}{|c|}{ Logístico } & \multicolumn{2}{|c|}{ Gompertz } & \multicolumn{2}{|c|}{ Richards } \\
\hline & $\mathrm{r}$ & $\mathrm{dm}$ & $\mathrm{r}$ & $\mathrm{dm}$ & $\mathrm{r}$ & $\mathrm{dm}$ & $\mathrm{r}$ & $\mathrm{Dm}$ & $\mathrm{r}$ & $\mathrm{dm}$ \\
\hline 0 & 0,03 & $-17,21$ & 0,07 & 13,14 & 0,11 & 31,04 & 0,09 & 23,44 & 0,39 & $-0,20$ \\
\hline 2 & 0,63 & 0,91 & 0,53 & 9,93 & 0,50 & 20,56 & 0,51 & 15,68 & 0,75 & 7,48 \\
\hline 4 & 0,82 & 3,94 & 0,79 & 4,03 & 0,74 & 10,56 & 0,76 & 7,52 & 0,86 & 7,03 \\
\hline 6 & 0,85 & $-1,34$ & 0,87 & $-8,81$ & 0,85 & $-7,61$ & 0,86 & $-8,35$ & 0,89 & $-3,89$ \\
\hline 8 & 0,78 & $-8,22$ & 0,82 & $-19,22$ & 0,82 & $-22,11$ & 0,82 & $-20,97$ & 0,82 & $-14,17$ \\
\hline 10 & 0,85 & $-3,56$ & 0,88 & $-15,59$ & 0,88 & $-21,17$ & 0,88 & $-18,70$ & 0,86 & $-10,57$ \\
\hline 12 & 0,85 & 6,39 & 0,86 & $-4,83$ & 0,87 & $-11,70$ & 0,86 & $-8,54$ & 0,83 & $-2,58$ \\
\hline 14 & 0,84 & 15,60 & 0,85 & 6,27 & 0,86 & $-0,68$ & 0,86 & 2,59 & 0,82 & 6,47 \\
\hline 16 & 0,82 & 18,47 & 0,83 & 11,42 & 0,83 & 5,26 & 0,83 & 8,26 & 0,75 & 11,02 \\
\hline 18 & 0,88 & 16,58 & 0,89 & 12,13 & 0,89 & 7,35 & 0,89 & 9,77 & 0,85 & 12,13 \\
\hline 20 & 0,90 & 10,76 & 0,91 & 8,79 & 0,91 & 5,68 & 0,91 & 7,33 & 0,87 & 7,69 \\
\hline 22 & 0,91 & 6,69 & 0,92 & 6,95 & 0,93 & 5,57 & 0,92 & 6,31 & 0,89 & 4,68 \\
\hline 24 & 0,91 & 6,08 & 0,92 & 8,34 & 0,92 & 8,61 & 0,92 & 8,49 & 0,88 & 4,38 \\
\hline 26 & 0,88 & 4,24 & 0,89 & 8,23 & 0,90 & 9,96 & 0,89 & 9,05 & 0,83 & 6,11 \\
\hline 28 & 0,88 & $-0,22$ & 0,90 & 5,24 & 0,90 & 8,20 & 0,90 & 6,66 & 0,81 & 3,32 \\
\hline 30 & 0,86 & $-11,58$ & 0,87 & $-5,16$ & 0,88 & $-1,24$ & 0,87 & $-3,17$ & 0,75 & $-6,28$ \\
\hline 32 & 0,89 & $-26,07$ & 0,90 & $-19,03$ & 0,90 & $-14,42$ & 0,90 & $-16,67$ & 0,82 & $-20,68$ \\
\hline 34 & 0,89 & $-37,28$ & 0,89 & $-29,82$ & 0,89 & $-24,79$ & 0,89 & $-27,27$ & 0,81 & $-32,70$ \\
\hline 36 & 0,77 & $-35,78$ & 0,79 & $-27,73$ & 0,79 & $-22,46$ & 0,79 & $-25,07$ & 0,70 & $-29,73$ \\
\hline 40 & 0,56 & 7,67 & 0,57 & 14,58 & 0,58 & 19,63 & 0,58 & 17,21 & 0,52 & 19,63 \\
\hline 44 & 0,63 & 6,61 & 0,64 & 12,84 & 0,64 & 17,26 & 0,64 & 15,17 & 0,64 & 19,71 \\
\hline 48 & 0,70 & 9,10 & 0,69 & 14,17 & 0,69 & 17,63 & 0,69 & 16,27 & 0,63 & 16,43 \\
\hline 52 & 0,59 & 14,81 & 0,60 & 18,63 & 0,60 & 21,00 & 0,60 & 19,88 & 0,52 & 18,98 \\
\hline 56 & 0,67 & $-4,12$ & 0,67 & $-1,22$ & 0,67 & 0,01 & 0,67 & $-0,50$ & 0,63 & $-3,72$ \\
\hline 60 & 0,72 & $-3,20$ & 0,71 & $-1,98$ & 0,70 & $-1,83$ & 0,71 & $-1,89$ & 0,65 & $-3,42$ \\
\hline 64 & 0,65 & 19,04 & 0,66 & 18,92 & 0,67 & 18,33 & 0,67 & 18,82 & 0,66 & 22,09 \\
\hline 68 & 0,67 & $-0,85$ & 0,67 & $-1,73$ & 0,68 & $-2,82$ & 0,68 & $-2,10$ & 0,62 & 0,30 \\
\hline 72 & 0,72 & $-2,81$ & 0,72 & $-4,54$ & 0,71 & $-6,23$ & 0,72 & $-5,11$ & 0,63 & $-7,61$ \\
\hline 76 & 0,66 & 12,99 & 0,66 & 10,58 & 0,66 & 8,41 & 0,66 & 9,37 & 0,66 & 11,05 \\
\hline 80 & 0,66 & $-6,07$ & 0,66 & $-9,24$ & 0,66 & $-11,86$ & 0,66 & $-10,61$ & 0,67 & $-8,86$ \\
\hline 84 & 0,68 & $-1,64$ & 0,68 & $-5,68$ & 0,67 & $-8,78$ & 0,68 & $-7,26$ & 0,64 & $-7,99$ \\
\hline 88 & 0,67 & 9,24 & 0,67 & 4,50 & 0,67 & 1,06 & 0,67 & 2,52 & 0,66 & 4,43 \\
\hline 92 & 0,71 & $-0,35$ & 0,71 & $-5,58$ & 0,71 & $-9,24$ & 0,71 & $-7,60$ & 0,73 & $-5,03$ \\
\hline 96 & 0,71 & $-5,83$ & 0,70 & $-11,65$ & 0,70 & $-15,56$ & 0,70 & $-13,86$ & 0,66 & $-12,72$ \\
\hline 100 & 0,59 & 11,14 & 0,58 & 4,83 & 0,58 & 0,71 & 0,58 & 2,30 & 0,59 & 6,17 \\
\hline 104 & 0,60 & $-1,38$ & 0,60 & $-8,13$ & 0,60 & $-12,44$ & 0,60 & $-10,28$ & 0,65 & $-7,90$ \\
\hline 108 & 0,68 & $-2,06$ & 0,67 & $-9,28$ & 0,67 & $-13,77$ & 0,67 & $-11,36$ & 0,66 & $-7,47$ \\
\hline
\end{tabular}

com todas as observações de todas as fêmeas, como um padrão médio de crescimento das fêmeas dessa raça. A escolha do modelo Bertalanffy implica a aceitação da existência de um ponto de inflexão nesta curva, por volta dos oito meses de idade.

\section{CONCLUSÕES}

1. O modelo Richards, com a parametrização adotada neste trabalho, não é indicado para estudos de crescimento da raça Guzerá, pois apresenta dificuldades computacionais para ajustamento.

2. Os modelos Brody, Bertalanffy, Logístico e Gompertz são adequados para descrever o crescimento em fêmeas da raça Guzerá.

3. O modelo Bertalanffy, com os parâmetros estimados no presente trabalho, é o mais indicado para representar a curva de crescimento média de fêmeas da raça Guzerá desta população, e também para ser utilizado em estudos do crescimento da raça. 


\section{REFERÊNCIAS}

ABASSA, K.P. Analysis of growth curve parameters of Gobra Zebu females in Senegal. Tropical Animal Health and Production, Dordrecht, v.19, p.223228, 1987.

BELTRAN, J.; BUTTS, W.; OLSON, T.; KOGER, M Growth patterns of two lines of Angus cattle selected using predicted growth parameters. Journal of Animal Science, Champaign, v.70, p.734-741, 1992.

BERTALANFFY, L. von. Quantitative laws in metabolism and growth. Quarterly Review of Biology, Chicago, v.32, p.217-230, 1957.

BRODY, S. Bioenergetics and growth. New York : Reinhold, 1945. 1023p.

BROWN, J.E.; FITZHUGH JUNIOR, H.A. CARTWRIGHT, T.C. A comparison of nonlinear models for describing weight-age relationships in cattle. Journal of Animal Science, Champaign, v.42, p.810-818, 1976.

CORTARELLI, A. Estudo da curva de crescimento de machos Nelore através de quatro modelos estocásticos. Jaboticabal : UNESP, 1973. 100p. Tese de Doutorado.

DENISE, R.S.K.; BRINKS, J.S. Genetic and environmenta aspects of the growth curve parameters in beef cows Journal of Animal Science, Champaign, v.61, p.1431-1440, 1985.

DUARTE, F.A.M. Estudo da curva de crescimento de animais da raça Nelore, através de cinco modelos estocásticos. Ribeirão Preto : Universidade de São Paulo, 1975. 284p. Tese de Livre Docência.

GOONEWARDENE, L.A.; BERG, R.T.; HARDIN, R.T A growth study of beef cattle. Canadian Journal of Animal Science, Ottawa, v.61, p.1041-1048, 1981.

HARTLEY, H.O. The modified Gauss-Newton method for the fitting of non-linear regression functions by least squares. Thechnometrics, Alexandria, v.3, p.269-280, 1961.

LAIRD, A.K. Dynamics of relative growth. Growth, Bar Harbor, v.29, p.249-263, 1965.

LÓPEZ DE TORRE, G.; CANDOTTI, J.J.; REVERTER, A.; BELLIDO, M.M.; VASCO, P.; GARCIA, L.J.; BRINKS, J.S. Effects of growth curve parameters on cow efficiency. Journal of Animal Science, Champaign, v.70, p.2668-2672, 1992.

LUDWIG, A. Ajustamento de curvas exponenciais ao crescimento de gado Nelore e análise de seus parâmetros. Viçosa : UFV, 1977. 84p. Dissertação de Mestrado.

MORROW, R.E.; McLAREN, J.B.; BUTTS, W.T. Effect of age on estimates of bovine growth-curve parameters. Journal of Animal Science, Champaign, v.47, p.352-357, 1978.

NADARAJAH, K.; MARLOWE, T.J.; NOTTER, D.R. Growth patterns of Angus Charolais, Charolais $\mathrm{x}$ Angus and Holstein $x$ Angus cows from birth to maturity. Journal of Animal Science, Champaign, v.59, p.957-966, 1984.

NELDER, J.A. The fitting of a generalization of the logistic curve. Biometrics, Washington, v.17, p.89-110, 1961.

PEREIRA, J.C.C. Melhoramento genético aplicado aos animais domésticos. Belo Horizonte : UFMG, 1983. 430p.

PEROTTO, D.; CUE, R.I.; LEE, A.J. Comparison of nonlinear functions for describing the growth curve of three genotypes of dairy cattle. Canadian Journal of Animal Science, Ottawa, v.72, p.773$782,1992$.

RICHARDS, F.J. A flexible growth function for empirical use. Journal of Experimental Botany, Oxford, v.10, p.290-300, 1959.

SAS INSTITUTE (Cary, Estados Unidos). SAS/STAT ${ }^{\text {TM: }}$ guide for personal computers, version 6. Cary, 1985. $387 \mathrm{p}$. 